\title{
SÍNDROME DE BURNOUT E ATUAÇÃO DO PROFISSIONAL DE EDUCAÇÃO FÍSICA EM CAPS-AD DA PARAÍBA
}

\section{BURNOUT SYNDROME AND PHYSICAL EDUCATION PROFESSIONAL PERFORMANCE IN PARAÍBA CAPS-AD}

\author{
Leonardo dos Santos Oliveira ${ }^{*}$, Valdir Andrade Tôrres Junior", Natália Maria Mesquita de Lima Quirino'
}

\begin{abstract}
Resumo. A Síndrome de Burnout (SB) é um fenômeno ocupacional que afeta milhares de profissionais de saúde no mundo inteiro, cuja prevalência tem sido analisada em diferentes contextos laborais. Contudo, a literatura não aponta trabalhos analisando a SB e os fatores associados à atuação de profissionais de Educação Física junto aos Centros de Apoio Psicossociais álcool e drogas (CAPSad). Portanto, este estudo investigou as dimensões da SB e suas relações com características da atuação do profissional de Educação Física em diferentes CAPS-ad da Paraíba. Em um estudo correlacional, profissionais de Educação Física, atuantes nos CAPS-ad de Cabedelo $(n=1)$, João Pessoa $(n=2)$, Monteiro $(n=1)$ e Sousa $(n=1)$, responderam um questionário autoaplicável composto por três seções: 1) características sociodemográficas; 2) características da atuação profissional; e 3) características de esgotamento profissional (Maslach Burnout Inventory - Human Services Survey). Após análise descritiva [frequências relativa e absoluta, média e desvio padrão ou mediana e intervalo interquartil (IQR)], o relacionamento entre as dimensões da SB e as variáveis relativas à atuação profissional foi verificado pelo coeficiente de Spearman e pelo teste Qui-quadrado. As dimensões de Exaustão Emocional (EE; 60,0\%) e Despersonalização (DP; 60,0\%) apresentaram baixa prevalência, contudo, observou-se alta prevalência para a dimensão Reduzida realização profissional (RP; 60,0\%). Foram constatados valores medianos de $18(\mathrm{IQR}=11), 6(\mathrm{IQR}=6)$ e $33(\mathrm{IQR}=18)$ pontos para $\mathrm{EE}, \mathrm{DP}$ e RP (ineficácia), respectivamente. A dimensão RP apresentou forte relacionamento com renda, tempo de profissão e carga horária total, mas, moderada com tempo de atuação no CAPS-ad. Não foram verificadas associações entre as dimensões da SB e a satisfação com o trabalho ( $\chi 2 \leq 3,06 ; P>0,05)$. Considerando a baixa prevalência da SB, os profissionais de Educação Física parecem demonstrar uma condição psicológica equilibrada para o atendimento de indivíduos com transtornos pelo uso de álcool e outras drogas.
\end{abstract}

PALAVRAS-CHAVE: Esgotamento Psicológico. Saúde Mental. Saúde do Trabalhador. Pessoal de Saúde.

Abstract. Burnout Syndrome (BS) is an occupational phenomenon that affects thousands of health professionals worldwide, whose prevalence has been widely analyzed in different work contexts. However, the literature does not indicate studies analyzing BS and the factors associated with the performance of Physical Education professionals at the Psychosocial Support Centers for alcohol and drugs (CAPS-ad). Therefore, this study investigated the BS and its relationship with the performance of the Physical Education professional at CAPS-ad in Paraíba. In a correlational study, Physical Education professionals, working at CAPS-ad from the cities of Cabedelo $(n=1)$, João Pessoa $(n=2)$, Monteiro $(n=1)$, and Sousa $(n=1)$, answered a questionnaire consisting of three sections: 1) sociodemographic characteristics; 2) characteristics of professional performance; and 3) characteristics of professional exhaustion (Maslach Burnout Inventory - Human Services Survey). After descriptive analysis [relative and absolute frequencies, mean and standard deviation or median and interquartile range (IQR)], the relationship between the dimensions of BS and the variables related to professional performance was verified by the Spearman coefficient and by the Chi-square test. The dimensions of Emotional Exhaustion (EE; 60.0\%) and Depersonalization (DP; 60.0\%) presented low prevalence, however, a high prevalence for the dimension of Reduced personal accomplishment was found (RPA; 60.0\%). Median values of $18(\mathrm{IQR}=11), 6(\mathrm{IQR}=6)$ and $33(\mathrm{IQR}=18)$ points were found for $\mathrm{EE}, \mathrm{DP}$, and $\mathrm{RPA}$ (ineffectiveness), respectively. The RPA dimension had a strong relationship with remuneration, time in the profession, and total workload, but a moderate correlation with time working at the CAPS-ad. There were no associations between the dimensions of BS and job satisfaction $(X 2 \leq 3.06 ; P>0.05)$. Due to low prevalence of $B S$, the Physical Education professionals seem to demonstrate a balanced psychological condition of the professionals for the care of individuals with disorders due to the use of alcohol and other drugs.

KEYWORDS: Burnout. Psychological. Mental Health. Occupational Health. Surveillance of the Workers Health. 


\section{INTRODUÇÃO}

A Síndrome de Burnout (SB) é um fenômeno ocupacional que afeta milhares de profissionais de saúde no mundo inteiro. ${ }^{1-3}$ Esse esgotamento é, geralmente, caracterizado por sentimentos de exaustão ou exaustão de energia, sentimentos de negativismo ou cinismo relacionados ao trabalho e eficácia profissional reduzida. ${ }^{3-5}$ Além disso, a SB é individual e se torna uma resposta crônica ao estresse interpessoal que existe e acontece no ambiente de trabalho. ${ }^{4,5}$ Portanto, um ambiente de trabalho desfavorável pode levar a problemas de ordem física e mental, absenteísmo, perda de produtividade, entre outros males. $^{3,4}$

Profissionais em esgotamento não estão simplesmente exaustos por elevada carga de trabalho. ${ }^{6}$ Há, da mesma forma, uma desconexão psicológica com seu trabalho, influenciando em sua motivação e identidade. ${ }^{4,6}$ No contexto laboral da saúde mental, ainda existe uma combinação de sentimentos que o trabalhador atuante nos cuidados de pessoas com transtornos mentais e/ou dependência química acaba vivenciando no ambiente de trabalho., 1,7,8

Estudos prévios com trabalhadores nos Centros de Apoio Psicossociais (CAPS), especializados em indivíduos com transtornos pelo uso de álcool e outras drogas (CAPS-ad), ainda apontam a necessidade de ampliação da equipe de saúde, problemas de diferenciação de contratos de trabalho e remuneração justa. 9,10

Assim como outros profissionais atuantes nos CAPS, o profissional de Educação Física (EF) também tem sua contribuição na restauração da saúde desses usuários. Nesta modalidade de CAPS, estes profissionais desenvolvem ações recreativas, oficinas terapêuticas, acolhimento, confraternizações, até as mais direcionadas à melhoria da aptidão física. ${ }^{11,12}$ Todavia, eles estão sujeitos a uma rotina ocupacional exaustiva, com diferentes tipos de agentes estressores, podendo desempenhar um trabalho depressivo e sem nenhuma satisfação ou motivação. ${ }^{13}$ Apesar de a SB ter sido amplamente analisada em diferentes contextos laborais, ${ }^{1,2,13,14}$ a literatura não aponta trabalhos analisando o fenômeno do esgotamento profissional e os fatores associados à atuação de profissionais de EF junto aos CAPS, sobretudo nos CAPSad.

O presente trabalho se torna importante em razão de que a SB vem sendo considerada um grande problema social, afetando de forma negativa a saúde de profissionais da área da saúde, dentre eles os profissionais de EF. Em adição, podese contribuir para oferecer novas ideias acerca do problema da SB, auxiliando na solução dos múltiplos desafios encontrados na relação entre pessoa e emprego. Portanto, este estudo investigou as dimensões da SB e suas relações com características da atuação do profissional de EF em diferentes CAPS-ad da Paraíba. Em face do exposto, a hipótese é que exista associação entre a SB e as características de atuação do profissional de EF nos CAPS-ad. 


\section{MATERIAL E MÉTODOS}

\section{Caracterização do estudo}

Tratou-se de um estudo com abordagem quantitativa (quantificando os dados obtidos para validar hipóteses), do tipo descritivo correlacional (descrevendo relações entre duas ou mais categorias, conceitos ou variáveis em um momento determinado). ${ }^{15}$

Constituem-se, como local da investigação, diferentes CAPS-ad da Rede de Atenção Psicossocial (RAPS) do estado da Paraíba, em outubro de 2020. À época, esta RAPS contemplava seis CAPS-ad em cada uma das cidades de Cabedelo, Cajazeiras, Campina Grande, Guarabira, Patos e Sousa, e nove CAPS-ad III localizados nas cidades de Campina Grande, João Pessoa (2 unidades), Mamanguape, Monteiro, Piancó, Pombal, Princesa Isabel e Sapé. ${ }^{16}$

\section{Participantes e aspectos éticos}

Participaram do estudo cinco profissionais de EF que trabalhavam em diferentes CAPS-ad da Paraíba, a saber: Cabedelo ( $n=1)$, João Pessoa $(n=2)$, Monteiro $(n=1)$ e Sousa $(n=1)$. O recrutamento dos participantes foi efetuado por meio de ligação telefônica para a Secretaria de Saúde do Estado da Paraíba e a Coordenação Estadual de Saúde Mental da Paraíba, solicitando-se o contato de cada CAPS-ad e, posteriormente, o contato desses profissionais.

Foi estabelecido o seguinte critério de inclusão: atuar como profissional de Educação Física em CAPS por, no mínimo, um ano. Foram excluídos os profissionais que não retornaram o questionário.

Este projeto foi aprovado pelo Comitê de Ética para Pesquisa com Seres
Humanos da FACENE (Protocolo: 264/2020 e CAAE: 36753320.9.0000.5179). Todos os profissionais consentiram virtualmente a participação voluntária no estudo a partir de um termo livre e esclarecido, conforme Resolução 466/12 do Conselho Nacional de Saúde.

\section{Instrumentos e procedimentos para coleta de dados}

Um questionário virtual (via Formulários Google) foi usado para coletar os dados, cujo tempo estimado para completar foi de 10-12 minutos. Este questionário foi composto por três seções:

- Características sociodemográficas: informações sobre faixa etária, sexo, raça, estado civil, número de filhos, remuneração (número de salários mínimos estimados em $\mathrm{R} \$$ 1045,00) e satisfação econômica foram coletadas por meio de um questionário elaborado pelos autores.

- Características da atuação profissional: informações sobre tempo de profissão, tempo de atuação no CAPS, carga horária semanal de trabalho no CAPS, turno de trabalho no CAPS, outros vínculos trabalhistas e satisfação com o trabalho foram coletadas por meio de um questionário elaborado pelos autores.

- Características de esgotamento profissional: aplicou-se a versão adaptada para a língua portuguesa do Maslach Burnout Inventory - Human Services Survey (MBI - HSS). ${ }^{17}$ Trata-se de um instrumento autoaplicável que contempla 
22 itens e analisa três dimensões da SB: exaustão emocional (EE), despersonalização (DP) e reduzida realização profissional (RP). A EE é descrita como um sentimento de ausência de energia física e emocional para realizar atividades profissionais. ${ }^{18}$ A DP é retratada como um afastamento emocional das outras pessoas, agindo de forma fria, com cinismo, ironia, falta de gentileza, tratando os outros como um objeto sem valor, levando, infelizmente, à falta de realização profissional e sentimento de incompetência. ${ }^{4,18}$ Por sua vez, a RP (ineficácia) é compreendida como um fenômeno paralelo e não necessariamente pertence ao problema de Burnout, que se manifesta em decorrência da desilusão e não satisfação com as tarefas ocupacionais. ${ }^{18-20}$ A cada um dos itens foram atribuídos graus de intensidade em uma escala do tipo Likert de 1 a 5: 1 (nunca); 2 (algumas vezes ao ano); 3 (algumas vezes ao mês); 4 (algumas vezes na semana); e 5 (diariamente). No geral, pode-se classificar a SB quando existem pontuações elevadas nas dimensões EE e DP com pontuação baixa na RP. ${ }^{21}$ Todavia, como critério diagnóstico de SB, após somatório das pontuações por dimensão do MBI-HSS, seguiu-se a classificação nos níveis alto, médio e baixo ${ }^{22}$ (Tabela 1).

\section{Análise estatística}

Dados de variáveis categóricas foram descritos por frequência absoluta (n) e relativa (\%). Por sua vez, dados de variáveis numéricas foram reportados por média e desvio padrão (DP) ou mediana (Md) e intervalo interquartil (IQR), respectivamente, para dados com distribuição normal ou não (Teste de ShapiroFrancia). A correlação entre os escores de cada dimensão da SB e as variáveis numéricas das características de atuação profissional foi obtida por meio do coeficiente Rho de Spearman, classificada conforme Dancey e Reidy. ${ }^{23}$ Por sua vez, a associação entre as dimensões da SB e satisfação com o trabalho foi verificada pelo teste Qui-quadrado $\left(\mathrm{X}^{2}\right)$ com ajuste de Fisher. A análise foi realizada por meio do programa IBM Statistical Package for the Social Sciences (SPSS), versão 25.0 (IBM Corp., Armonk, EUA). Um valor-P menor que $5 \%$ foi considerado estatisticamente significante.

TABELA 1. Dimensões da Síndrome de Burnout pelo Maslach Burnout Inventory (MBI-HSS).

\begin{tabular}{lcccc}
\hline \multirow{2}{*}{\multicolumn{1}{c}{ Dimensões }} & \multicolumn{3}{c}{ Pontuações } & \multirow{2}{*}{ Questões } \\
\cline { 2 - 4 } & Baixa & Média & Alta & (19 \\
Exaustão emocional & $<6$ & $19-26$ & $\geq 27$ & $1,2,3,6,8,13,14,16$ e 20 \\
Despersonalização & $26-9$ & $\geq 10$ & $5,10,11,15$ e 22 \\
Reduzida realização profissional (ineficácia) & $\geq 40$ & $34-39$ & $\geq 33$ & $4,7,9,12,17,18,19$ e 21 \\
\hline \hline
\end{tabular}

\section{RESULTADOS E DISCUSSÃO}

Dos 15 locais de pesquisa, apenas dois não dispunham de profissional de EF à época. Dos 13 profissionais convidados para o estudo, oito foram excluídos pelo não retorno do questionário. Portanto, a pesquisa envolveu apenas cinco participantes (39,4\% do total), possivelmente em virtude da pandemia de COVID-19, em que muitos serviços estavam 
paralisados. Estes profissionais apresentaram média de idade de $37(\mathrm{DP}=9)$ anos, sendo a maioria do sexo feminino $(60,0 \%)$, brancos (80,0\%), casados (60,0\%), com 1 a 2 filhos, com média de remuneração de $3(D P=1)$ salários mínimos e com $60,0 \%$ de insatisfação com sua situação econômica.

Quanto às características de atuação profissional, verificou-se que a média do tempo de profissão foi de $13(\mathrm{DP}=11)$ anos, a média do tempo de atuação nos CAPSad foi de $3(\mathrm{DP}=2)$ anos e a média da carga horária semanal junto aos CAPS-ad foi de 18 $(D P=15)$ horas. Além disso, os profissionais trabalhavam nos turnos matutino ou diurno, com 80,0\% deles apresentando vínculo externo (ex.: professor em escola ou academia) com média de carga horária de, aproximadamente, 10 horas. Apenas 20,0\% dos profissionais de EF estavam insatisfeitos com o trabalho.

A Tabela 2 resume as características de esgotamento profissional, cujas prevalências foram baixas para EE $(60,0 \%)$ e DP $(60,0 \%)$, mas, alta para RP $(60,0 \%)$. Além disso, foram constatados valores medianos de 18 (IQR= $11), 6(I Q R=6)$ e $33(I Q R=18)$ pontos para as dimensões EE, DP e RP, respectivamente.

TABELA 2. Escore e classificação dos resultados do Maslach Burnout Inventory (MBI-HSS) entre os profissionais de Educação Física (EF) atuantes em CAPS-ad da Paraíba ( $n=5)$. Paraíba, Brasil, 2020.

\begin{tabular}{cccc}
\hline Profissional de EF & EE & DP & RP \\
\hline \hline 1 & 15 (Baixa) & 8 (Baixa) & 38 (Média) \\
$\# 2$ & 19 (Média) & 6 (Média) & 33 (Alta) \\
$\# 3$ & 31 (Alta) & 14 (Alta) & 30 (Alta) \\
$\# 4$ & 18 (Baixa) & 5 (Baixa) & 39 (Média) \\
$\# 5$ & 14 (Baixa) & 5 (Baixa) & 11 (Alta) \\
\hline \hline & Cada profissional foi descrito por um “\#”; EE, exaustão emocional; DP, despersonalização; RP, \\
& & reduzida realização pessoal (ineficácia).
\end{tabular}

A Tabela 3 reporta a análise correlacional entre características de atuação profissional e dimensões da SB. A dimensão EE apresentou relacionamento moderado com tempo de profissão e tempo de atuação no CAPS-ad. A dimensão DP apresentou moderado relacionamento com a carga horária semanal no CAPS- ad. A dimensão RP apresentou fortes relacionamentos com remuneração, tempo de profissão e carga horária total, mas, moderada correlação com tempo de atuação no CAPS-ad e carga horária semanal no CAPS-ad. Em adição, não foram verificadas associações entre as dimensões da SB e a satisfação com o trabalho ( $\chi 2 \leq 3,06 ; \mathrm{P}>0,05)$.

TABELA 3. Análise correlacional entre características de atuação profissional e dimensões da Síndrome de Burnout (SB) ( $n=5)$. Paraíba, Brasil, 2020.

\begin{tabular}{lccc}
\hline \multirow{2}{*}{ Características de atuação profissional } & \multicolumn{3}{c}{ Dimensões da SB } \\
\cline { 2 - 4 } & EE & DP & RP \\
\hline Remuneração & $0,000(P=1,000)$ & $-0,148(P=0,812)$ & $0,866(P=0,058)$ \\
Tempo de profissão & $-0,400(P=0,505)$ & $-0,205(P=0,741)$ & $0,800(P=0,104)$ \\
Tempo de atuação no CAPS & $0,500(P=0,391)$ & $-0,359(P=0,553)$ & $0,400(P=0,505)$ \\
Carga horária semanal no CAPS & $-0,103(P=0,870)$ & $0,400(P=0,511)$ & $0,462(P=0,434)$ \\
Carga horária total & $0,000(P=1,000)$ & $0,154(P=0,810)$ & $\mathbf{0 , 9 0 0}(P=\mathbf{0 , 0 4 0})$ \\
\hline
\end{tabular}


Em geral, tanto os escores quanto a prevalência em cada dimensão, observados para os profissionais de EF atuantes em diferentes CAPS-ad da Paraíba, são menores quando comparados a trabalhadores da área da saúde mental. ${ }^{1}$ Dado que a $\mathrm{EE}$ é, tipicamente, considerada uma dimensão central da SB, 4,5 este estudo aponta apenas um profissional com SB (\#3), o que representa uma prevalência de $20,0 \%$. Contudo, os altos escores de RP podem indicar produtividade ou capacidade reduzida destes trabalhadores, bem como incapacidade de lidar com situações no trabalho. ${ }^{5}$ Portanto, diante da suposição explícita de que as pessoas podem experimentar diferentes padrões de esgotamento, que podem mudar com o tempo, ${ }^{24}$ é preciso ficar atento ao adoecimento desta classe trabalhadora.

Analisando separadamente as dimensões da SB, a alta pontuação para a RP pode estar atrelada a um possível desânimo com a profissão escolhida. ${ }^{19,20}$ Apesar de os profissionais de EF apresentarem experiência profissional acima de 10 anos, eles tinham pouca vivência nos CAPS-ad, o que pode justificar o elevado escore na RP uma vez que quanto maior tempo de profissão, menor tendência à SB, pelo motivo de já ter findado o estágio em que são frequentes as decepções profissionais. Além disso, a RP pode estar relacionada a más condições de trabalho, satisfação com a supervisão até o conteúdo que o trabalho oferece. Como consequência, estes profissionais podem apresentar queda no desempenho ao atenderem este público.

No contexto dos profissionais de EF de diferentes CAPS-ad da Paraíba, a RP se desenvolveu em paralelo às demais dimensões de Burnout, ao invés de sequencialmente. ${ }^{4,24}$ Uma vez que EE e DP parecem emergir da presença de sobrecarga de trabalho e conflitos sociais, ${ }^{4}$ nosso estudo aponta novas direções ao modelo de fase de Burnout, em que o cinismo é a fase inicial mínima, seguida pelos acréscimos de ineficácia e, por fim, de exaustão. $^{24}$ Este achado pode auxiliar na solução de problemas relacionados à atuação do profissional de EF neste ambiente de trabalho, haja vista que sua prática difere, com efeito, dos demais profissionais da saúde. ${ }^{12,25}$

As fortes correlações da RP com remuneração, tempo de profissão e carga horária total não são consistentes com outras pesquisas, ${ }^{1}$ mas, parecem refletir um perfil ineficaz no trabalho. ${ }^{24} \mathrm{O}$ aprofundamento desta discussão requer análises estatísticas que vão além da análise correlacional realizada, contudo, em virtude da quantidade de participantes, não foi possível efetuá-las. Ao assumir esta limitação, sugere-se que novas investigações incluam mais profissionais e considerem as análises de perfis latentes, ainda que este procedimento seja específico para uma determinada amostra. ${ }^{24}$

$O$ fato de os profissionais de EF apresentarem pontuações baixa e média, tanto para EE, quanto para DP, respectivamente, está em desacordo com achados recentes. ${ }^{1,5}$ Curiosamente, a carga horária deles não se apresentou vinculada à exaustão ou ao cinismo. Este achado implica em relativo fator de proteção, pois, sabe-se da vulnerabilidade que a maioria dos profissionais da saúde apresenta nos serviços de saúde mental, nos quais o cuidado ao tratar indivíduos com sofrimento psíquico, por si, já é um fator estressor que pode levar ao adoecimento do trabalhador. ${ }^{1,7}$ Portanto, apesar de não se ter verificado a SB na maioria dos profissionais investigados, é importante avaliar e aplicar mudanças resolutivas para que, futuramente, eles não venham a apresentar quadros de esgotamento profissional.

Uma informação que merece destaque foi que embora os profissionais de EF tenham reportado insatisfação com questões salariais, observou-se elevada satisfação com o trabalho. É bem conhecido que estar satisfeito com o que a instituição oferece, com os benefícios e 
políticas organizacionais é fundamental para a prevenção da SB. ${ }^{7,21,26}$ Diferentes estudos também têm apontando que a satisfação no trabalho está associada ao apoio de colegas e supervisores e menor pressão de carga de trabalho. ${ }^{27,28}$ Todavia, uma exploração mais aprofundada sobre os aspectos específicos de cada equipe de trabalho se faz necessária, especialmente, no estado da Paraíba. ${ }^{16}$

Os profissionais de EF são, historicamente, uma categoria com múltiplos conflitos relacionados à precarização e à frustação na carreira profissional. ${ }^{29}$ Este fato pode explicar a forte correlação entre a carga horária e a dimensão relacionada à ineficácia profissional, de modo que parece haver uma confusão de sentimentos próprios dos profissionais de EF, comparado ao padrão de relacionamento conhecido para outros profissionais de saúde que trabalham na área

\section{CONCLUSÃO}

Considerando a baixa prevalência da SB, os profissionais de Educação Física de diferentes CAPS-ad da Paraíba parecem demonstrar uma condição psicológica equilibrada para o atendimento de indivíduos com transtornos pelo uso de álcool e outras drogas. Além disso, apesar de os participantes

\section{AGRADECIMENTOS}

Agradecemos à Coordenação Estadual de Saúde Mental da Paraíba, na pessoa de laciara Mendes, aos participantes da saúde mental. ${ }^{1,7}$ No contexto dos CAPS, os profissionais de EF ainda não exercem plena funcionalidade conforme proposto em resolução especializada. ${ }^{30}$ Nessa perspectiva, aspectos organizacionais podem ter uma influência persistente e de longo alcance, especialmente, quando violam as expectativas básicas de justiça e equidade. ${ }^{4}$

Como recomendação para estudos futuros, sugere-se a análise de uma maior quantidade de características de atuação profissional, bem como um aumento no número de participantes, a fim de reduzir a probabilidade de erro do tipo II. Destacase que a presente investigação não é representativa do panorama dos CAPS-ad da Paraíba, mas, fornece algum direcionamento para as autoridades competentes no cuidado desta classe trabalhadora.

terem reportado elevada carga horária semanal, inclusive com vínculo externo ao CAPS-ad, no geral, as características de atuação profissional apresentaram vínculo estatístico forte apenas com a dimensão relacionada à ineficácia profissional.

pela disponibilidade em colaborar e ao Prof. Josélio Soares de Oliveira Filho pela revisão técnica do manuscrito. 


\section{REFERÊNCIAS BIBLIOGRÁFICAS}

1. O'Connor K, Neff DM, Pitman S. Burnout in mental health professionals: A systematic review and meta-analysis of prevalence and determinants. Eur Psychiatry. 2018;53:74-99.

2. Güler Y, Şengül S, Çaliş H, Karabulut Z. Burnout syndrome should not be underestimated. AMB Rev Assoc Med Bras. 2019;65(11):1356-60.

3. OPAS/OMS. CID: burnout é um fenômeno ocupacional [Internet]. Washington, DC: OPAS/OMS; 2019. [cited 19 mai 2020]. Available from: https:// www.paho.org/bra/.

4. Maslach C, Schaufeli WB, Leiter MP. Job burnout. Annu Rev Psychol. 2001;52:397-422.

5. Maslach C, Leiter MP. Understanding burnout: New models. In: Cooper CL, Quick JC, editors. The handbook of stress and health: A guide to research and practice. Chichester: John Wiley \& Sons Ltd.; 2017. p. 36-56.

6. Mendez I, Martinez-Ramon JP, Ruiz-Esteban C, Garcia-Fernandez JM. Latent Profiles of Burnout, Self-Esteem and Depressive Symptomatology among Teachers. Int J Environ Res Public Health. 2020;17(18).

7. Morse G, Salyers MP, Rollins AL, Monroe-DeVita $M$, Pfahler C. Burnout in mental health services: $A$ review of the problem and its remediation. Adm Policy Ment Health. 2012;39(5):341-52.

8. Brotto TCA, Dalbello-Araujo M. É inerente ao trabalho em saúde o adoecimento de seu trabalhador? Rev Bras Saúde Ocup. 2012;37(126):290305 .

9. Clodoaldo SS, Barbosa GC, Oliveira MAF. Satisfação dos trabalhadores de um centro de atenção psicossocial em álcool e outras drogas. Rev Uningá. 2017;52(1):12-7.

10. Silva EA, Costa II. O profissional de referência em
Saúde Mental: das responsabilizações ao sofrimento psíquico. Rev Latinoam Psicopatol Fundam. 2010;13(4):635-47.

11. Costa TM, Santos FM, Silva RCB, Oliveira HLR, Ilha PV, Graup S. Educação Física e saúde mental: atuação profissional nos Centros de Atenção Psicossocial. Pensar Prát. 2017;20(3):539-51.

12. Furtado RP, Neto RC, Rios GB, Martinez JFN, de Oliveira MFM. Educação física e saúde mental: uma análise da rotina de trabalho dos profissionais dos CAPS de Goiânia. Movimento (ESEFID/UFRGS). 2016;22(4):1077-90.

13. Guedes D, Gaspar E. "Burnout” em uma amostra de profissionais de Educação Física brasileiros. Rev Bras Educ Fís Esporte. 2016;30(4):999-10.

14. Silva ABN, Maximin DAFM, Souto CGV, Virgínio NA. Síndrome de Burnout em profissionais de enfermagem na Unidade de Terapia Intensiva. Rev Ciênc Saúde Nova Esperança. 2016;14(1):79-86.

15. Sampieri H, Collado F, Lucio B. Metodologia de

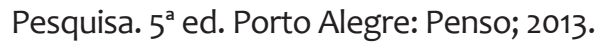

16. PARAÍBA. Relatório do monitoramento dos serviços da Rede de Atenção Psicossocial (RAPS) da Paraíba em 2018.2. João Pessoa: Secretaria de Estado da Saúde; Gerência Executiva de Atenção à Saúde; Coordenação Estadual de Saúde Mental SES/PB, 2020.

17. Lautert L. O desgaste profissional: estudo empírico com enfermeiras que trabalham em hospitais. Rev Gaucha Enferm. 1997;18(2):133.

18. Benevides-Pereira AMT, Machado PGB, PortoMartins PC, Carrobles JA, Siqueira JO. Confirmatory factor analysis of the ISB-Burnout Syndrome Inventory. Psychology, Community \& Health. 2017;6(1):28-41.

19. Maslach C. What have we learned about burnout 
and health? Psychol Health. 2001;16(5):607-11.

20. Schaufeli WB, Bakker AB. Job demands, job resources, and their relationship with burnout and engagement: A multi-sample study. J Organiz Behav. 2004;25(3):293-315.

21. Maslach C. Finding solutions to the problem of burnout. Consult Psychol J: Pract Res. 2017;69(2):143.

22. Moreira DS, Magnago RF, Sakae TM, Magajewski FRL. Prevalência da síndrome de burnout em trabalhadores de enfermagem de um hospital de grande porte da Região Sul do Brasil. Cad Saude Publica. 2009;25(7):1559-68.

23. Dancey C, Reidy J. Statistics without maths for psychology. $7^{\mathrm{a}}$ ed. New York: Pearson; 2017.

24. Leiter MP, Maslach C. Latent burnout profiles: A new approach to understanding the burnout experience. Burn Res. 2016;3(4):89-100.

25. Furtado RP, Sousa MF, Martinez JFN, Rabelo NS, Oliveira NSR, Simon WJ. Desinstitucionalizar o cuidado e institucionalizar parcerias: desafios dos profissionais de Educação Física dos CAPS de
Goiânia em intervenções no território. Saude Soc. 2017;26(1):183-95.

26. Maslach C, Leiter MP. New insights into burnout and health care: Strategies for improving civility and alleviating burnout. Med Teach. 2017;39(2):160-63. 27. Scanlan JN, Still M. Job satisfaction, burnout and turnover intention in occupational therapists working in mental health. Aust Occup Ther J. 2013;60(5):310-18.

28. Luther L, Gearhart T, Fukui S, Morse G, Rollins AL, Salyers MP. Working overtime in community mental health: Associations with clinician burnout and perceived quality of care. Psychiatr Rehabil J. 2017;40(2):252-59.

29. Furtado RP, Santiago LP. Educação Física e trabalho: considerações a respeito da inserção profissional de egressos da FEF-UFG. Rev Bras Educ Fís Esporte. 2015;29(2):325-36.

30. Conselho Federal de Educação Física. Resolução CONFEF $n^{\circ}$ 230/2012. Dispõe sobre Especialidade Profissional em Educação Física na área de Saúde Mental. Diário Oficial da União. 2012. p. 175. 